\title{
Clock Distribution Networks in 3-D Integrated Systems
}

\author{
Vasilis F. Pavlidis, Member, IEEE, Ioannis Savidis, and Eby G. Friedman, Fellow, IEEE
}

\begin{abstract}
D integration is an important technology that addresses fundamental limitations in on-chip interconnects. Several design issues related to $3-\mathrm{D}$ circuits, such as multiplane synchronization, however, need to be addressed. A comparison of three 3-D clock distribution network topologies is presented in this paper. Good agreement is shown between the modeled and experimental results of a 3-D test circuit composed of three device planes. Successful operation of the $3-\mathrm{D}$ test circuit at $1.4 \mathrm{GHz}$ is demonstrated. Clock skew, clock delay, signal slew, and power dissipation measurements for the different clock topologies are also provided. The measurements suggest that each topology provides certain advantages and disadvantages in terms of different performance criteria. The proper choice, consequently, of a clock distribution network is not dictated by a single design objective but rather by the overall 3-D system design requirements including availability of resources and number of bonded planes.
\end{abstract}

Index Terms-3-D clock characterization, 3-D clock distribution, 3-D clock modeling, 3-D synchronization.

\section{INTRODUCTION}

A $\mathrm{N}$ OMNIPRESENT and challenging issue for synchronous digital circuits is the reliable distribution of the clock signal to the many hundreds of thousands of sequential elements distributed throughout a synchronous circuit [1], [2]. The complexity of this task is further exacerbated in 3-D integrated circuits (ICs) as sequential elements belonging to the same clock domain (i.e., synchronized by the same clock signal) can be located on multiple planes. Another fundamental issue in the design of clock distribution networks is low power consumption, since the clock network dissipates a significant portion of the total power consumed by a synchronous circuit [3], [4]. This constraint is stricter for 3-D ICs due to the higher power density and related thermal concerns.

Manuscript received December 14, 2009; revised April 22, 2010 and July 28, 2010; accepted August 11, 2010. Date of publication October 14, 2010; date of current version October 28, 2011. This work was supported in part by the National Science Foundation under Contract CCF-0541206, Contract CCF-0811317, and Contract CCF-0829915, by grants from the New York State Office of Science, Technology \& Academic Research to the Center for Advanced Technology in Electronic Imaging Systems, and by grants from Intel Corporation, Eastman Kodak Company, and Freescale Semiconductor Corporation.

V. F. Pavlidis is with the Integrated Systems Laboratory, EPFL, Lausanne, CH-1015, Switzerland (e-mail: vasileios.pavlidis@epfl.ch)

I. Savidis and E. G. Friedman are with the Department of Electrical and Computer Engineering, University of Rochester, Rochester, NY 14627 USA (e-mail: iosavid@ece.rochester.edu; friedman@ece.rochester.edu).

Color versions of one or more of the figures in this paper are available online at http://ieeexplore.ieee.org.

Digital Object Identifier 10.1109/TVLSI.2010.2073724

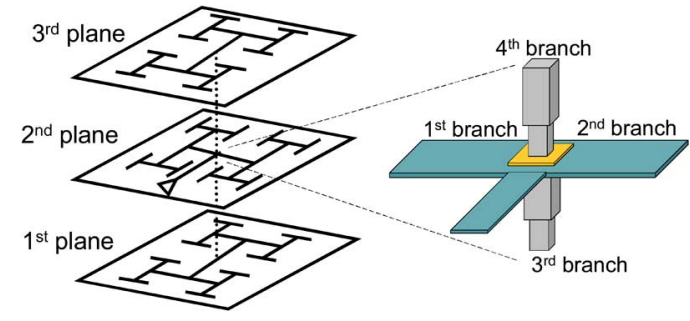

Fig. 1. Schematic of a 3-D clock tree. The magnified detail illustrates the four branches that emanate from the output of the clock driver in the second plane. Two branches propagate the clock signal in the second plane, while the vertical TSVs transfer the clock signal to the first and third planes.

In 2-D circuits, symmetric interconnect structures, such as $\mathrm{H}$ and $\mathrm{X}$-trees, are widely utilized to distribute the clock signal across a circuit [2]. The symmetry of these structures permits the clock signal to arrive at the leaves of the tree at approximately the same time, resulting in synchronous data processing. Maintaining this symmetry within a 3-D circuit, however, is a difficult task.

An extension of an $\mathrm{H}$-tree to three dimensions does not guarantee equidistant interconnect paths from the root to the leaves of the tree. The clock signal propagates through vertical interconnects, typically implemented by through silicon vias (TSVs) from the output of the clock driver to the center of the H-tree on the other planes. The impedance of the TSVs can increase the time for the clock signal to arrive at the leaves of the tree on these planes as compared to the time for the clock signal to arrive at the leaves of the tree located on the same plane as the clock driver. Furthermore, in a multiplane 3-D circuit, three or four branches can emanate at each branch point, as depicted in Fig. 1. The third and fourth branches propagate the clock signal to the other planes within the 3-D circuit. Similar to a design methodology for a 2-D H-tree topology, the width of each branch is reduced by a third (or more) of the segment width preceding the branch point to match the impedance at that branch point [2]. This requirement, however, is difficult to achieve as the third and fourth branches are connected through a TSV.

Global signaling issues in 3-D circuits, such as clock signal distribution, have only recently been explored [5]-[9]. Recent studies consider thermal effects on buffered 3-D clock trees [10] and H-tree topologies [11], [12]. No experimental characterization of 3-D clock distribution networks, however, has yet been presented. Measurements from a 3-D test circuit employing several clock distribution architectures are presented in this paper. The test circuit was fabricated by the MIT Lincoln Laboratories (MITLL) [13], [14].

The objective of the paper is to summarize the analysis of different 3-D clock distribution topologies for both skew (and 


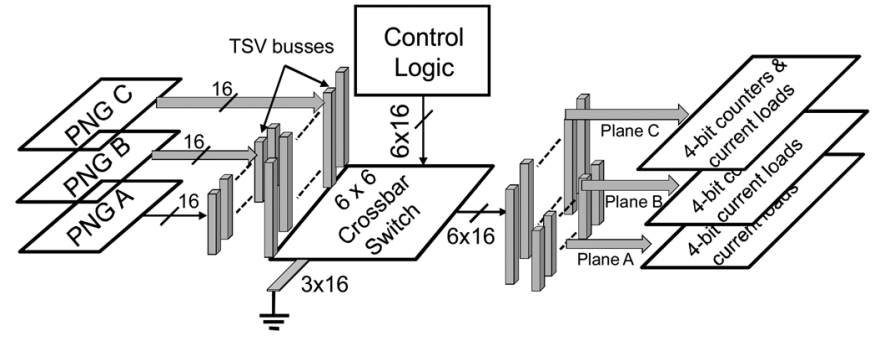

Fig. 2. Block diagram of the logic circuit for each clock topology.

therefore delay) and power consumption. Clock distribution networks of increasing asymmetry in a 3-D stack are also investigated. Analysis of the skew and power consumption provides enhanced understanding of the advantages and disadvantages of each topology, and aides in the design of the synchronous circuitry in 3-D integrated systems. In addition, the effect of the TSVs on distributing the clock signal within a stack of device planes is described. The effect that these structures have on propagating clock signals in a fabricated 3-D circuit is demonstrated and described for the first time in this paper.

In the following section, the design of the 3-D test circuit is described. A brief discussion of the MITLL process is provided in Section III. Experimental results and a discussion of the characteristics of the three clock distribution networks are presented in Section IV. Simulations of the clock distribution topologies including expressions modeling the 3-D via impedance are compared to experimental results in Section V. Some conclusions are offered in Section VI. The closed-form expressions characterizing the impedance of the 3-D via are provided in Appendix A, and the circuit parameters used to model the clock skew within the 3-D clock topologies are summarized in Appendix B.

\section{Design of the 3-D Test Circuit}

The test circuit consists of three blocks. Each block includes the same logic circuit but implements a different clock distribution architecture. The total area of the test circuit is $3 \mathrm{~mm}$ $\times 3 \mathrm{~mm}$, where each block occupies an approximate area of 1 $\mathrm{mm}^{2}$. Each block contains about 30,000 transistors with a power supply voltage of $1.5 \mathrm{~V}$. The design kit used for the implementation has been developed by North Carolina State University. ${ }^{1}$ The common logic circuitry within each clock module is described in Section II-A, and the different clock distribution architectures are reviewed in Section II-B.

\section{A. 3-D Circuit Architecture}

The logic circuit common to the three blocks is described in this section. An overview of the logic circuitry is depicted in Fig. 2. The function of the logic is to emulate different switching patterns of the circuit and operating conditions for the clock distribution networks under investigation. The logic is repeated in each plane and includes three pseudorandom number generators (PNG), a six-by-six bit crossbar switch, control logic for the crossbar switch, several groups of four-bit counters, and current loads.

${ }^{1}$ [Online]. Available: http://www.ece.ncsu.edu/erl/3DIC/pub

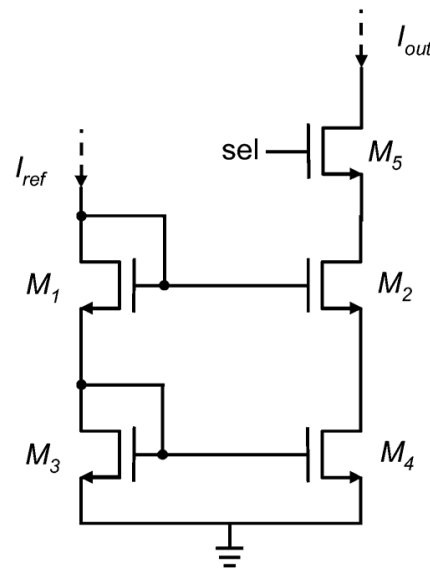

Fig. 3. Cascoded current mirror with an additional control transistor, M5.

The pseudorandom number generators use linear feedback shift registers and XOR operations to generate a random 16-bit word every clock cycle once the generators are initialized [15]. The data flow in this circuit can be described as follows. After resetting the circuit, the PNG are initialized and the control logic connects each input port to the appropriate output port. Since the control logic includes an eight-bit counter, each input port of the crossbar switch is successively connected every 256 clock cycles to each output port.

The output ports of the crossbar switch, each 16 bits wide, are connected to four 4-bit counters (see Fig. 2). Each of these counters is loaded with a four-bit word, counts upwards, and is loaded with a new word each time all four bits are equal to one. The MSB of each counter is connected to four current loads that are turned on when this bit is equal to one. Since the counters are loaded with random numbers through the crossbar switch, the current loads draw a variable amount of current during circuit operation. This randomness is used to mimic different switching patterns that can exist within a circuit.

The current loads are implemented with cascode current mirrors, as shown in Fig. 3. The reference current $I_{\mathrm{ref}}$ is externally provided to control the amount of current drawn from the circuit. The gate of transistor M5 is connected to the MSB of a four-bit counter, shown in Fig. 3 as the sel signal. This additional device is used to switch the current sinks. The width of the devices shown in Fig. 3 is $W_{1}=W_{2}=W_{3}=W_{4}=$ $600 \mathrm{~nm}$ and $W_{5}=2000 \mathrm{~nm}$.

Several TSVs are used to connect these circuits. For example, in each block, each PNG is placed on a different plane but at the same location within each plane. Vertical busses connect the output of the PNGs to the input ports of the switch. Additionally, interplane signals connect the current loads with the control signal, which is generated by the MSB of the 4-bit counters. Furthermore, the reset signal is distributed by the TSVs to each sequential element throughout the multiple planes.

Several capacitors are included in each circuit block and serve as an extrinsic decoupling capacitance which is implemented by MIM capacitors [16]. Additionally, each of the circuit blocks is supplied by separate power and ground pads (three pairs of power and ground pads per block) to ensure that each block can be individually tested. Furthermore, one pair of power and 


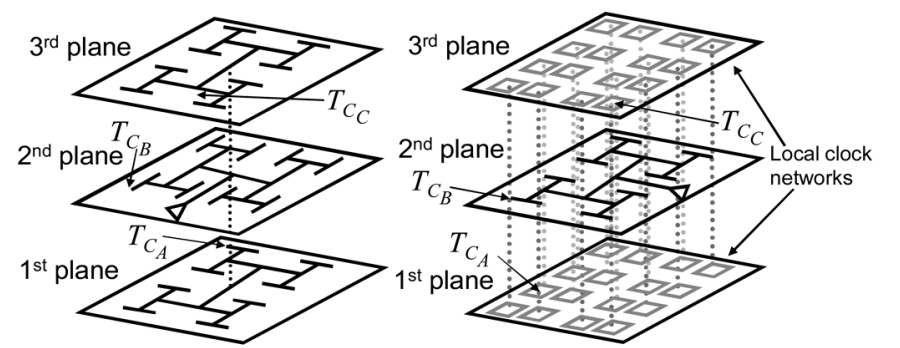

(a)

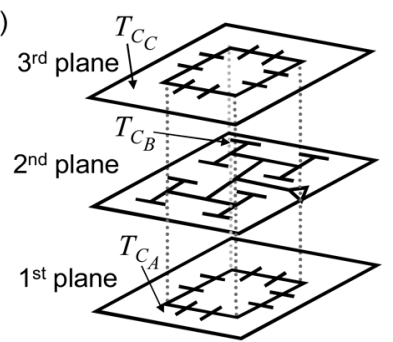

(b)

Fig. 4. Three 3-D clock distribution networks within the test circuit: (a) H-trees, (b) H-tree and local rings, and (c) H-tree and global rings.

ground pads is connected to the pad ring to provide protection from electrostatic discharge and provide power and ground to the $\mathrm{I} / \mathrm{O}$ drivers.

\section{B. 3-D Clock Topologies}

Several clock network topologies for 3-D ICs are described in this section. These architectures combine different topologies which are commonplace in 2-D circuits, such as H-trees, rings, and meshes [2]. Each of the three blocks includes a different clock distribution structure, which is schematically illustrated in Fig. 4. The dashed lines depict vertical interconnects implemented by groups of through silicon vias. Multiple TSVs at the connection points between the clock networks are used to lower the resistance of the vertical path while enhancing reliability.

As shown in Fig. 4, these topologies range from purely symmetric to highly asymmetric networks to investigate the different features of these topologies. A primary objective is to determine the effect of the TSVs on the clock skew. The symmetry of the H-tree topology should be sufficient if the effect of the TSVs is small (for this specific technology). Alternatively, load balancing the global rings may reduce the delay of the clock signal caused by the TSVs. Local meshes may be preferable since the distribution of the clock signal to the sinks is primarily limited within a physical plane. Stacks of TSVs subsequently connect the sinks on other planes through local rings. This topology offers the advantage of limiting most of the clock paths within one physical plane, while distributing the signal vertically to localized areas within neighboring planes.

The effect that these topological choices have on the clock skew, power dissipation, and signal slew are experimentally investigated. Since the clock signal is distributed in three dimensions, achieving equidistant signal propagation in a 3-D system is not straightforward. This task is further complicated by the different impedance characteristics of the vertical and horizontal interconnects. Consequently, the objective is to provide a global clock topology that produces sufficiently low skew (or predictable skew for delay compensation) within

(intra-plane) and among (inter-plane) the planes of a 3-D circuit. The symmetry of an H-tree and the load balancing characteristics of rings and meshes are thereby exploited. Additionally, the power consumed by each 3-D clock architecture is considered due to the importance of thermal issues in 3-D circuits.

In each of the circuit blocks, the clock driver for the entire clock network is located on the second plane. The location of the clock driver is chosen to ensure that the clock signal propagates through identical vertical interconnect paths to the first and third planes, ideally resulting in the same delay. The clock driver is implemented with a traditional chain of tapered buffers [17], [18]. Additionally, buffers are inserted at the leaves of each $\mathrm{H}$-tree in all three topologies. The width of the branches within the H-tree is halved at each branch point [19], with an initial width of $8 \mu \mathrm{m}$.

Note that in a 3-D circuit employing an even number of planes, the inherent symmetry of an H-tree topology in the vertical direction is not possible, increasing the inter-plane clock skew between specific planes, as depicted in Fig. 4. Therefore, for a 3-D technology supporting $n$ physical planes, where $n$ is even, symmetry along the vertical direction is not feasible. Placing the clock driver in plane $n / 2+1$ or $n / 2-1$ results in an increase in skew between the first and $n$th plane equal to the effective delay of the group of TSVs connecting the two successive planes. This increase in delay can be compensated by placing fewer TSVs (with a higher effective impedance) in the vertical direction.

The architectures employed in the blocks are as follows.

Block A: All of the planes contain a four level H-tree (i.e., equivalent to 16 leaves) with identical interconnect characteristics. All of the H-trees are connected through a group of TSVs at the output of the clock driver. Note that in Fig. 4(a) the H-tree on the second plane is rotated by $90^{\circ}$ with respect to the H-trees on the other two planes. This rotation eliminates inductive coupling between the H-trees. All of the H-trees are shielded with two parallel lines connected to ground.

Block B: A four level H-tree is included in the second plane. All of the leaves of this H-tree are connected by four TSVs to small local rings on the first and third planes, as illustrated in Fig. 4(b). As in Block A, the H-tree is shielded with two parallel lines connected to ground. Additional interconnect resources form local rings. Due to the limited interconnect resources, however, achieving a uniform mesh in each ring is difficult. Clock routing is constrained by the power and ground lines as only three metal layers are available on each plane [13], [14].

Block $C$ : The clock distribution network for the second plane is a shielded four level H-tree. Two global rings are utilized for the other two planes, as shown in Fig. 4(c). Buffers are inserted to drive each ring, which are connected by TSVs to the four branch points on the second level of the $\mathrm{H}$-tree. The rings on planes $\mathrm{A}$ and $\mathrm{C}$ are connected to the second level of the H-tree for two reasons; first, to avoid an unnecessarily long ring that would result in a significant capacitive load, and second, to maintain a ring with sides of equal length. Additionally, connecting the ring to 


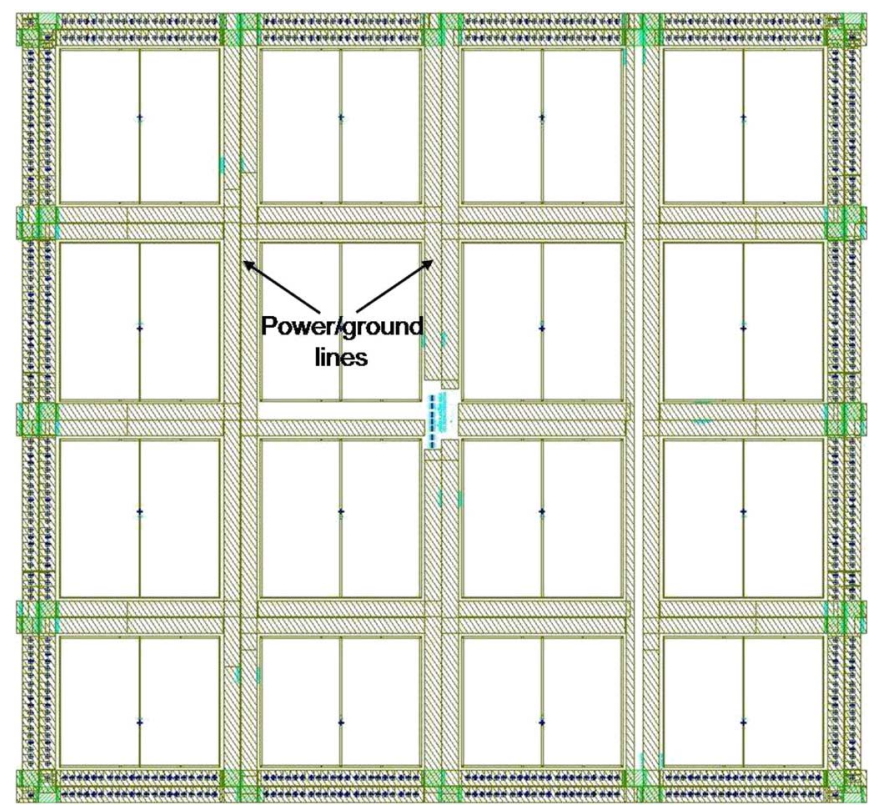

Fig. 5. Power distribution network for the local ring topology.

the leaves at the perimeter of the H-tree results in a considerable difference in the load among the sinks of the tree, since only the outer leaves are connected to the ring. The registers in each plane are connected either directly to the rings on the first and third planes or are driven by buffers at the leaves of the H-tree on the second plane. With this arrangement, the balancing properties of the rings results in low interplane skew for the first and third planes. In addition, since the interplane path to these planes is the same for both planes, the skew between these two planes is low as compared to the H-tree topology in the second plane.

A primary objective of this paper is to evaluate the delay and power characteristics of different clock distribution architectures. A secondary and related objective is to analyze the characteristics of asymmetric topologies in 3-D systems. This objective poses several limitations on the power distribution network within each block. Power and ground rings at the periphery of each block are utilized. Although this architecture is not optimal, the structure is sufficiently small $\left(\sim 1 \mathrm{~mm}^{2}\right)$. The small size of the blocks does not cause a significant voltage drop across and among the planes. In addition, minimal $L d i / d t$ noise is observed during circuit operation. The power and ground rings on each plane are connected by a large number of TSVs to lower the impedance of the vertical interconnections. The local rings topology requires greater area for distributing power and ground in the first and third planes, where local rings distribute the clock signal. The resulting clock and power networks for planes $\mathrm{A}$ and $\mathrm{C}$ are illustrated in Fig. 5 for this specific block. In this topology, the power distribution network consists of a coarse mesh of power and ground lines [16].

\section{FABRICATION OF THE 3-D TEST CIRCUIT}

The manufacturing process developed by MITLL for fully depleted silicon-on-insulator (FDSOI) 3-D circuits is summarized here [13], [14]. The MITLL process is a wafer level 3-D

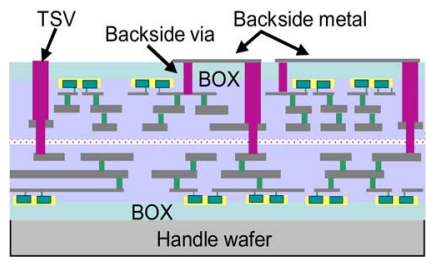

(a)

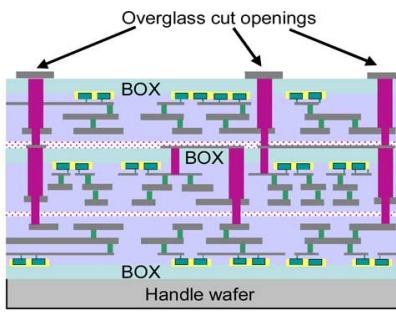

(b)
Fig. 6. Cross-section of a 3-D circuit based on the MITTLL process: (a) an intermediate step and (b) the fully fabricated 3-D stack [13]. The second plane is flipped and bonded with the first plane, while the third plane is bonded face-toback with the second plane. The backside metal layer and vias and the through silicon vias are also shown.

integration technology with up to three FDSOI wafers bonded to form a 3-D circuit. The diameter of the wafers is $150 \mathrm{~mm}$. The minimum feature size of the devices is $180 \mathrm{~nm}$, with one polysilicon layer and three metal layers interconnecting the devices on each wafer. A backside metal layer also exists on the upper two planes, providing the starting and landing pads for the TSVs, and the I/O, power supply, and ground pads for the entire 3 -D circuit. An attractive feature of this process is the high density TSVs. The dimensions of these vias are $1.75 \mu \mathrm{m} \times 1.75 \mu \mathrm{m}$, much smaller than the through silicon via in many existing 3-D technologies [20], [21]. An intermediate step of the fabrication process is illustrated in Fig. 6, where some salient features of this technology are also depicted. SOI technologies are particularly suitable for 3-D circuits, since the SOI device layers can be used for both monolithic [22] and wafer level 3-D integrated systems. In the latter case, SOI is a better solution for 3-D circuits because it is possible to aggressively etch the wafers as compared to standard bulk CMOS technologies [23]. This situation is due to the high selectivity of the etching solutions, where a $\mathrm{Si}$ to $\mathrm{SiO}_{2}$ selectivity of $300: 1$ is possible [23]. This capability results in significantly shorter through silicon vias, a critical component in 3-D systems. The primary obstacle for 3-D SOI technologies is the high thermal resistance of the oxide which impedes the heat removal process.

As depicted in Fig. 6, this process includes both face-to-face and face-to-back plane bonding. The TSV length, however, is not affected due to the aggressive etching feasible with this technology. Alternatively, employing a bulk CMOS technology can require TSVs of longer length due to the greater thickness of the silicon substrate [24]. In the context of clock skew, the presence of TSVs with different lengths increases the asymmetry in the vertical direction, requiring more careful design to balance the clock signal delay across the plane. The horizontal interconnect is partitioned into segments by the TSVs, which also affects the clock signal delay.

\section{EXPERIMENTAL RESULTS}

The clock distribution network topologies of the 3-D test circuit are evaluated in this section. The fabricated circuit is depicted in Fig. 7(a), where the individual blocks can be distinguished. A magnified view of one block is shown in Fig. 7(b). Each block includes four RF pads for measuring the delay of the clock signal. The pad located at the center of each block provides the input clock signal. The clock input waveform is a 
TABLE I

Clock SKew Among the Planes of Each Block

\begin{tabular}{|c|c|c|c|c|c|c|c|c|}
\hline \multirow{2}{*}{$\begin{array}{c}\text { Clock } \\
\text { distribution } \\
\text { network }\end{array}$} & \multirow{2}{*}{$\begin{array}{c}\text { Clock skew } \\
(\mathrm{ps})\end{array}$} & \multicolumn{8}{|c|}{ Frequency $(\mathrm{MHz})$} \\
\cline { 3 - 10 } & & 10 & 20 & 40 & 80 & 160 & 500 & 1000 \\
\hline \hline \multirow{2}{*}{$\begin{array}{c}\text { H-tree } \\
\text { (Fig. 4a) }\end{array}$} & $t_{B A}=\left|t_{B}-t_{A}\right|$ & 59.0 & - & 59.7 & 91.4 & - & 18.7 & 26.2 \\
\cline { 2 - 10 } & $t_{B C}=\left|t_{B}-t_{C}\right|$ & 66.8 & - & 88.5 & 62.5 & - & 20.1 & 30.2 \\
\cline { 2 - 10 } & $t_{A C}=\left|t_{A}-t_{C}\right|$ & 7.8 & - & 28.8 & 28.9 & - & 1.4 & 4.1 \\
\hline \multirow{2}{*}{$\begin{array}{c}\text { Local rings } \\
\text { (Fig. 4b) }\end{array}$} & $t_{B A}=\left|t_{B}-t_{A}\right|$ & 122.5 & - & 82.3 & - & 70.0 & 159.1 & 112.1 \\
\cline { 2 - 10 } & $t_{B C}=\left|t_{B}-t_{C}\right|$ & 70.8 & - & 48.9 & - & 38.0 & 144.5 & 131.1 \\
\cline { 2 - 9 } & $t_{A C}=\left|t_{A}-t_{C}\right|$ & 51.7 & - & 33.4 & - & 32.0 & 14.5 & 19.1 \\
\hline \multirow{2}{*}{$\begin{array}{c}\text { Global rings } \\
\text { (Fig. 4c) }\end{array}$} & $t_{B A}=\left|t_{B}-t_{A}\right|$ & - & 28.9 & 23.6 & 31.0 & 30.4 & - & 68.3 \\
\cline { 2 - 9 } & $t_{B C}=\left|t_{B}-t_{C}\right|$ & - & 41.0 & 38.2 & 36.7 & 42.2 & - & 18.6 \\
\cline { 2 - 9 } & $t_{A C}=\left|t_{A}-t_{C}\right|$ & - & 12.2 & 14.6 & 5.7 & 11.8 & - & 49.7 \\
\hline \multirow{2}{*}{}
\end{tabular}

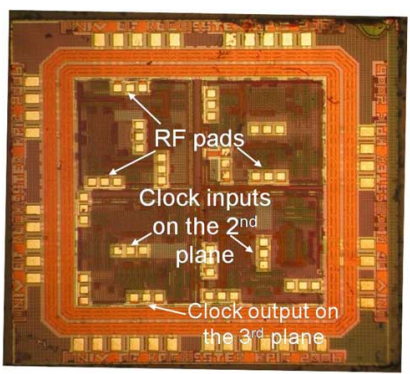

(a)

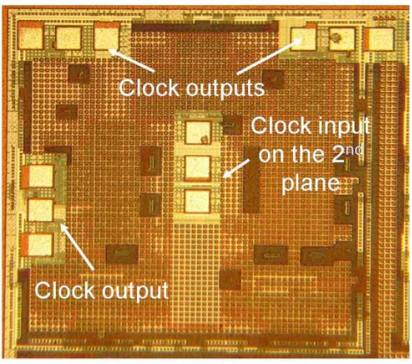

(b)

Fig. 7. Fabricated 3-D circuit: (a) all of the blocks and dc pads and (b) a magnified view of one block.

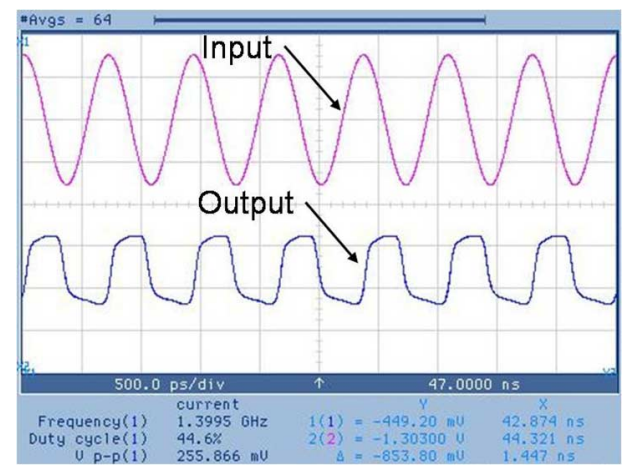

Fig. 8. Clock signal input and output waveform from the topology illustrated in Fig. 4(c).

sinusoidal signal with a dc offset, which is converted to a square waveform at the output of the clock driver. The remaining three RF pads are used to measure the delay of the clock signal at specific points on the clock distribution network within each plane. A buffer is connected at each of these measurement points. The output of this buffer drives the gate of an open drain transistor connected to the RF pad.

A clock waveform acquired from the topology combining an $\mathrm{H}$-tree and global rings is illustrated in Fig. 8, demonstrating circuit operation at $1.4 \mathrm{GHz}$. The clock skew between the planes of each block is listed in Table I. The delay of the clock signal from the RF input pad at the center of each block to the measurement point on plane $i$ is denoted as $t_{i}$ in Table I. For example, $t_{A}$ denotes the delay of the clock signal to the measurement point on plane A. The clock delay from the source node on the second plane to each leaf on the three separate planes is listed in Table II. Differences between the data listed in Tables I
TABLE II

Measured Clock Delay From the Root to the Leaves of EACH PLANE FOR EACH BLOCK

\begin{tabular}{|c|c|c|c|}
\hline Clock distribution & \multicolumn{3}{|c|}{ Clock delay (ns) } \\
\cline { 2 - 4 } network & $t_{A}$ & $t_{B}$ & $t_{C}$ \\
\hline \hline H-trees (Fig. 4a) & 0.359 & 0.338 & 0.325 \\
\hline Local rings (Fig. 4b) & 0.445 & 0.350 & 0.300 \\
\hline Global rings (Fig. 4c) & 0.530 & 0.480 & 0.445 \\
\hline
\end{tabular}

and II are due to the method of analysis for the clock skew and clock delay, respectively. The clock skew between each leaf is the average skew as determined from data samples collected at $10,20,40,80,160,500$, and $1000 \mathrm{MHz}$. The clock delay is the average root to leaf delay from data collected at frequencies of 500 and $1000 \mathrm{MHz}$. The difference in the delay of the clock signal between two measurement points on planes $i$ and $j$ is notated as $t_{i j}$.

For the H-tree topology, the clock signal delay is measured from the root to a leaf of the tree on each plane, with no additional load connected to these leaves. The skew between the leaves of the $\mathrm{H}$-tree on planes $\mathrm{A}$ and $\mathrm{C}$ (i.e., $t_{\mathrm{AC}}$ ) is effectively the delay of a stacked TSV traversing the three planes transferring the clock signal from the target leaf to the RF pad on the third plane (plane C). A schematic of this topology including a path of the clock signal is shown in Fig. 9. The delay of the clock signal to the sink of the $\mathrm{H}$-tree on the second plane $t_{B}$ is larger due to the additional capacitance coupled to that quadrant of the $\mathrm{H}$-tree. This capacitance is intentional on-chip decoupling capacitance placed under the quadrant, increasing the measured skew of $t_{B C}$ and $t_{B A}$. This topology produces, on average, comparable skew to the global ring topology, and less skew than the local rings clock structure.

The measured and average slew for each block is reported in Table III. The measurements are for a clock frequency of $1 \mathrm{GHz}$, where the time resolution is sufficiently small to produce reasonable accuracy $\left(t_{\mathrm{step}}=1.22 \mathrm{ps}\right)$. From the reported results, any undershoots during the falling edge increase significantly as compared to during the rise time. The mismatch between the size of the devices in the clock buffers also contributes to unbalanced clock edges, although from simulations, equal rise and fall times are demonstrated.

In the $\mathrm{H}$-tree topology, each leaf of a tree is connected to only those registers located within the same plane. Allowing one sink of an $\mathrm{H}$-tree to drive a register on another plane adds the delay of another TSV to the clock signal path, further increasing the 
TABLE III

Slew Measurements of the Investigated Clock Distribution Networks

\begin{tabular}{|c|c|c|c|c|c|c|c|c|}
\hline \multirow{2}{*}{ Topology } & \multicolumn{3}{|c|}{ Rise time $(20 \%$ to $80 \%)(\mathrm{ps})$} & \multicolumn{3}{c|}{ Fall time (80\% to 20\%) (ps) } \\
\cline { 2 - 9 } & Plane A & Plane B & Plane C & Avg. & Plane A & Plane B & Plane C & Avg. \\
\hline \hline H-trees & 92.5 & 98.3 & 164.2 & 118.3 & 116.9 & 194.7 & 210.0 & 173.9 \\
\hline Local rings & 91.0 & 79.1 & 127.3 & 99.1 & 85.7 & 90.6 & 284.5 & 153.6 \\
\hline Global rings & 108.7 & 101.3 & 108.4 & 106.1 & 104.0 & 59.3 & 83.8 & 82.4 \\
\hline
\end{tabular}

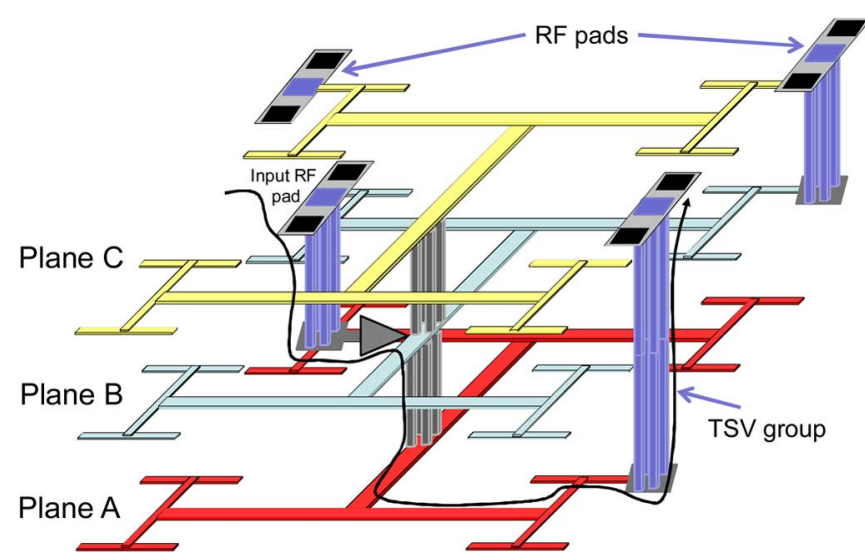

Fig. 9. 3-D H-tree topology, where the path of the clock signal from the input to plane A and through a group of TSVs to an output RF pad is shown (not to scale).

delay. Consequently, the registers within each plane are connected to the $\mathrm{H}$-tree on the same plane. Note that this approach does not require the data paths to be within one plane.

The clock skew among the planes is greater for the local ring topology as compared to the H-tree and global ring topologies, primarily due to the imbalance in the clock load for certain local rings. Indeed, this topology has only 16 tap points within the global clock distribution network; three times fewer than the H-tree topology illustrated in Fig. 4(a). This difference can produce a considerable load imbalance, greatly increasing the local clock skew as compared to the local clock skew within the $\mathrm{H}$-tree and global ring topologies. By inserting the local rings on planes $\mathrm{A}$ and $\mathrm{C}$, connected to the 16 sinks of the $\mathrm{H}$-tree on the second plane, the local clock skew is significantly larger than either the $\mathrm{H}$-tree only, or $\mathrm{H}$-tree with global ring topologies.

Consequently, a limitation of the local rings topology is that greater effort is required to control the local skew. The fewer number of sinks driven by the global clock distribution network increases the number of registers clocked by each sink. To better explain this situation, consider a segment of each topology, as shown in Figs. 10(a) and (b), respectively. For the $\mathrm{H}$-tree topology, the clock signal is distributed from three sinks, one on each plane, to the registers within the circular area depicted in Fig. 10(a). Note that the radius of the circle on planes $\mathrm{A}$ and $\mathrm{C}$ is slightly smaller to compensate for the additional delay of the clock signal due to the impedance characteristics of the TSVs. The registers located within these regions satisfy specific local skew constraints. Alternatively, in the case of the local ring topology, the clock signal at the sinks of the H-tree on the second plane feeds registers on each of the three planes. Consequently, each sink of the tree connects to a larger number of registers as compared to the H-tree topology, as depicted by

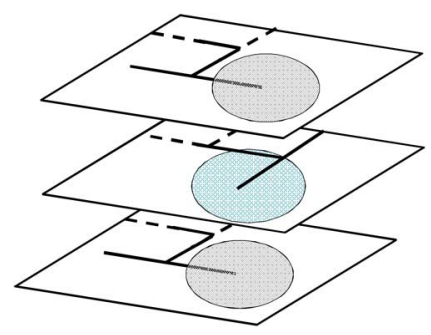

(a)

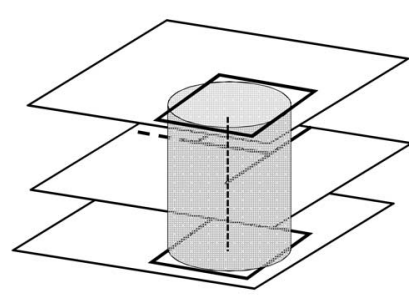

(b)
Fig. 10. Part of the clock distribution networks illustrated in Fig. 4(a) and (b). (a) The local clock skew is individually adjusted within each plane of the $\mathrm{H}$-tree topology. (b) The local skew is simultaneously adjusted for all of the planes within the local ring topology.

the shaded region in Fig. 10(b). Despite the beneficial effect of the local rings, load imbalances are more pronounced with this topology. Alternatively, the H-tree topology [see Fig. 4(a)] utilizes a significant amount of interconnect resources, dissipating greater power.

The clock distribution network with the global rings exhibits low skew for planes $\mathrm{A}$ and $\mathrm{C}$, those planes that include the global rings. The objective of this topology is to evaluate the effectiveness of a less symmetric architecture in distributing the clock signal within a 3-D circuit. Although the clock load on each ring is non-uniformly distributed, the load balancing characteristic of the rings yields a relatively low skew between the planes. Since the clock distribution network on the second plane is implemented with an H-tree, the skew between adjacent planes is significantly larger than the skew between the top and bottom planes. Note that the sinks of the H-tree on plane B are located at a greater distance from the rings on planes $\mathrm{A}$ and $\mathrm{C}$ [see Fig. 4(c)]. A combination of H-tree and global rings, consequently, is not a suitable approach for 3-D circuits due to the difficulty in matching the distance that the clock signal traverses on each plane from the sink of the tree or the ring to the many registers distributed across a plane.

The measured power consumption of the blocks operating at $1 \mathrm{GHz}$ is reported in Table IV. The local ring topology dissipates the lowest power. This topology requires the least interconnect resources for a global clock network, since the local rings are connected at the output of the buffers located on the last level of the H-tree on the second plane. In addition, this topology requires a small amount of local interconnect resources as compared to the H-tree and global rings topologies. Most of the registers are connected directly to the local rings. Alternatively, the power consumed by the H-tree topology is the highest, as this topology requires three $\mathrm{H}$-trees and additional wiring for the local connections to the leaves of each tree. In addition, the largest number of buffers is included in this topology. This number is threefold as compared to the number of buffers used 
TABLE IV

Measured Power Consumption of Each Block Operating at $1 \mathrm{GHz}$

\begin{tabular}{|c|c|}
\hline Clock distribution network & Power consumption (mW) \\
\hline H-trees (Fig. 4a) & 260.3 \\
\hline Local rings (Fig. 4b) & 168.3 \\
\hline Global rings (Fig. 4c) & 228.5 \\
\hline
\end{tabular}

for the local ring topology. Finally, the global rings block consumes slightly less power than the H-tree topology due to the reduced amount of wiring resources used by the global clock network.

Although the local ring topology requires the least interconnect resources, a large number of TSVs is required for the interplane connections. Since the TSVs block all of the metal layers and occupy silicon area, the routing blockage increases considerably as compared to the H-tree topology. The global rings topology requires a moderate number of TSVs as only four connections between the vertices of the rings and the branch points of the H-tree are necessary.

Since 3-D integration greatly increases the complexity of designing an integrated system, a topology that offers low overhead during the design process of a 3-D clock distribution network is preferable. From this perspective, a potential advantage of the H-tree topology is that each plane can be individually analyzed. This approach is supported by the $\mathrm{H}$-tree topology since the clock distribution network in each plane is exclusively connected to registers within the same plane. Alternatively, in the local ring topology, registers from all of the planes, which are connected to each sink of the tree on the second plane, all need to be simultaneously considered.

\section{Models of the Clock Distribution Network}

TOPOLOGIES INCORPORATING THE 3-D VIA IMPEDANCE

Simulation of the fabricated clock distribution topologies incorporating the modeled electrical impedance of the interplane $3-\mathrm{D}$ vias is described in this section. A comparison between the simulated and experimental results is also presented here. The electrical impedance of the 3-D vias is described for several diameters, lengths, dielectric thicknesses (bulk), and via-to-via spacings [25], [26]. The extracted parameters are used in the closed-form expressions characterizing the 3 -D via impedance [26]. These equations are used here to model the contribution of the 3-D vias to the delay and skew characteristics of the clock distribution topologies and are summarized in Appendix A.

In addition to characterizing the electrical parameters of the TSVs, the electrical characteristics of the clock distribution network on each plane are determined through numerical simulation. This set of simulations has been performed for the three widths used in the fabricated test circuit, and for five different lengths. Trend lines for the capacitance, dc resistance, $1 \mathrm{GHz}$ resistance, dc self- and mutual inductance, and the asymptotic self- and mutual inductance $f_{\text {asym }}$ approximate the electrical parameters of different length interconnect segments within the clock network. These simulations include two ground return paths spaced $2 \mu \mathrm{m}$ from either side of the clock line. These return paths behave as ground for the electrical field lines emanating from the clock line, resulting in a more accurate estimate of the capacitance.
The electrical paths of the clock signal propagating from the root to the leaves of each plane for the H-tree clock topology [see Fig. 4(a)] is depicted in Fig. 11. The size of the source follower nMOS transistor and the dimensions of the clock buffers at the root, leaves, and output circuitry are included in Appendix B. The clock network on each plane is composed of $50 \mu \mathrm{m}$ segments, where a $\pi$-model represents the electrical properties of each segment. These $50 \mu \mathrm{m}$ segments model the distributive electrical properties of the interconnect. Similarly, when either meshes [see Fig. 4(b)] or rings [see Fig. 4(c)] are used on planes A and C (see Fig. 11), each $50 \mu \mathrm{m}$ segment is replaced with an equivalent $\pi$-model to more accurately represent the single mesh and ring structure within the test circuit. Note that for the mesh structures, the clock signal is distributed to planes $\mathrm{A}$ and $\mathrm{C}$ from the leaves of the $\mathrm{H}$-tree in plane $\mathrm{B}$ while for the rings topology, the clock signal distributed to planes A and $\mathrm{C}$ is driven by buffers at the second level of the H-tree. The delay from the root to the leaves of each plane is included in Table V.

The clock delays listed in Table $\mathrm{V}$ are compared with the measured values listed in Table II. Good agreement between the model and experimental data is shown. The per cent error between the model and experimental clock delays is listed in Table VI. A maximum error of less than $10 \%$ is achieved for the clock paths within the H-tree topology. The larger errors shown in Table VI are due to the small time scale being examined. All of the values listed in Tables II and V are less than $550 \mathrm{ps;}$ therefore, any small deviation in delay produces a large error.

\section{CONCLUSION}

The design of a clock distribution network for application to 3-D circuits is considerably more complex than the design of a 2-D clock distribution network. Three topologies to globally distribute a clock signal within a 3-D circuit have been evaluated. A 3-D test circuit, based on the MITLL 3-D IC manufacturing process, has been designed, fabricated, and measured and is shown to operate at $1.4 \mathrm{GHz}$. Clock skew simulations incorporating both numerical simulation and analytic expressions produce comparable results to the experimentally extracted clock skew measurements. The clock skew measurements indicate that a topology combining the symmetry of an $\mathrm{H}$-tree on the second plane and global rings on the remaining two planes results in low clock skew in 3-D circuits while consuming a moderate amount of power. This structure, however, produces the largest root to leaf clock delay as compared to the other investigated topologies. Alternatively, for the H-tree and local rings topology, the lowest power is consumed. The performance characteristics of these topologies suggest that the target requirements should be considered when designing a 3-D clock distribution network.

\section{APPENDIX A}

\section{EleCtrical Parameters of A THrough Silicon Via}

The resistance of a $3-D$ via [26] is

$$
R_{\mathrm{DC}}=\frac{1}{\sigma_{W}} \frac{\mathfrak{L}}{\pi \mathfrak{R}^{2}}
$$




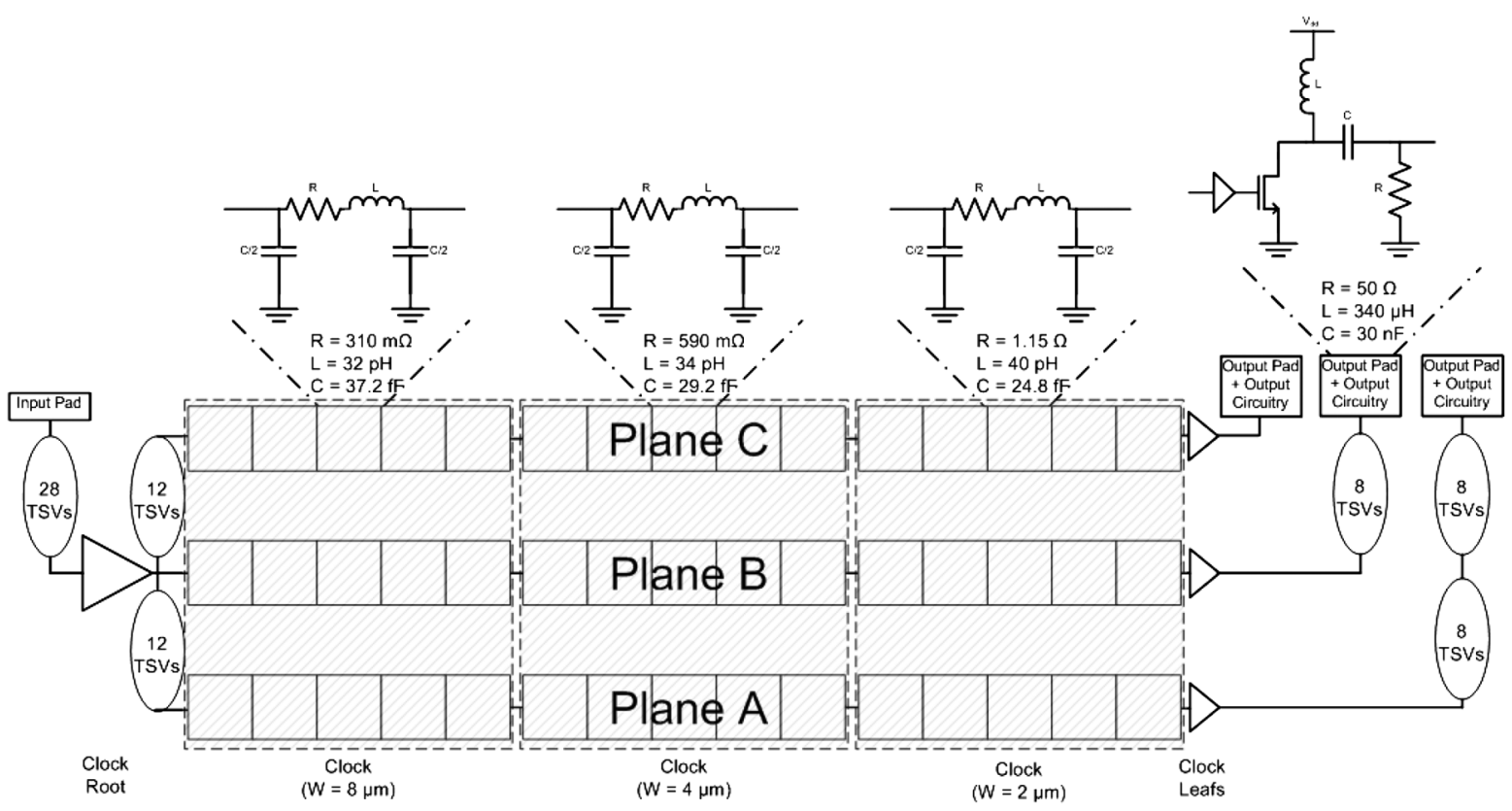

Fig. 11. Structure of clock distribution network path from Fig. 4(a) to model clock skew. The number within each TSV oval represents the number of parallel TSVs between device planes.

TABLE V

Modeled Clock Delay From the Root to the LEAVES OF EACH PLANE FOR EACH BLOCK

\begin{tabular}{|c|c|c|c|}
\hline \multirow{2}{*}{$\begin{array}{c}\text { Clock distribution } \\
\text { network }\end{array}$} & \multicolumn{3}{|c|}{ Clock delay (ns) } \\
\cline { 2 - 4 } & $t_{A}$ & $t_{B}$ & $t_{C}$ \\
\hline \hline H-trees (Fig. 4a) & 0.359 & 0.355 & 0.351 \\
\hline Local rings (Fig. 4b) & 0.325 & 0.323 & 0.321 \\
\hline Global rings (Fig. 4c) & 0.340 & 0.295 & 0.272 \\
\hline
\end{tabular}

TABLE VI

Per Cent Error Between Modeled and Experimental Clock Delay

\begin{tabular}{|c|c|c|c|}
\hline \multirow{2}{*}{$\begin{array}{c}\text { Clock distribution } \\
\text { network }\end{array}$} & \multicolumn{3}{|c|}{ Clock delay \% error } \\
\cline { 2 - 4 } & $t_{A}$ & $t_{B}$ & $t_{C}$ \\
\hline \hline H-trees (Fig. 4a) & 0 & -4.9 & -7.4 \\
\hline Local rings (Fig. 4b) & 36.9 & 8.4 & -6.5 \\
\hline Global rings (Fig. 4c) & 1.5 & 5.7 & 9.1 \\
\hline
\end{tabular}

$$
R_{1 \mathrm{GHz}}= \begin{cases}\alpha \frac{1}{\sigma_{W}} \frac{\mathfrak{L}}{\pi\left[\mathfrak{R}^{2}-(\mathfrak{R}-\delta)^{2}\right]}, & \text { if } \delta<\mathfrak{R} \\ \alpha \frac{1}{\sigma_{W}} \frac{\mathfrak{L}}{\pi \mathfrak{R}^{2}}, & \text { if } \delta \geq \mathfrak{R}\end{cases}
$$

where $\mathfrak{L}$ and $\mathfrak{R}$ are the TSV length and radius, respectively, and $\sigma_{W}$ is the conductivity of tungsten. The effect of the skin depth $\delta$ is included in (2), which reduces the cross-sectional area of the 3-D via. An empirical constant $\alpha$ is used to fit the $1 \mathrm{GHz}$ resistance to the simulation data. The equations for $\delta$ and $\alpha$ are provided in (4)-(6), respectively,

$$
\begin{aligned}
& \delta= \frac{1}{\sqrt{\pi f \mu_{o} \sigma_{W}}} \\
& \alpha=\left\{\begin{array}{c}
0.0472 D_{\mu m}^{0.2831} \ln \left(\frac{\mathfrak{L}}{D}\right) \\
+2.4712 D_{\mu m}^{-0.269}, \\
0.0091 D_{\mu m}^{1.0806} \ln \left(\frac{\mathfrak{L}}{D}\right) \\
+1.0518 D_{\mu m}^{0.092},
\end{array} \quad \text { if } \delta \geq \mathfrak{R} .\right.
\end{aligned}
$$

For frequencies other than dc and $1 \mathrm{GHz}$, the values produced by (1)-(3) are adjusted by (7)

$$
R_{f_{\text {new }}}=\left(R_{1 G H z}-R_{\mathrm{DC}}\right) \sqrt{\frac{f_{n e w}}{f_{1 \mathrm{GHz}}}}+R_{\mathrm{DC}}
$$

The inductance of a 3-D via is described by (8)-(11) [26]. These four equations express the self- $\left(L_{11}\right)$ and mutual inductance $\left(L_{21}\right)$ of the vias at both dc and high frequency. The expressions for the high frequency inductance represent the asymptotic value of the inductance. The range of frequencies for which the closed-form inductance expressions are valid is depicted in Fig. 12. The dc and high frequency self-inductance of a TSV is described by (8) and (9), respectively, while the dc and high frequency mutual inductance of a TSV is described by (10) and (11), respectively,

$$
\begin{aligned}
& L_{11}=\alpha \frac{\mu_{o}}{2 \pi}\left[\ln \left(\frac{\mathfrak{L}+\sqrt{\mathfrak{L}^{2}+\mathfrak{R}^{2}}}{\mathfrak{R}}\right) \mathfrak{L}\right. \\
& \left.+\mathfrak{R}-\sqrt{\mathfrak{L}^{2}+\mathfrak{R}^{2}}+\frac{\mathfrak{L}}{4}\right] \text {, for DC } \\
& L_{11}=\alpha \frac{\mu_{o}}{2 \pi}\left|\ln \left(\frac{2 \mathfrak{L}}{\mathfrak{R}}\right) \mathfrak{L}-1\right| \text {, for } f_{\text {asym }} \\
& L_{21}=\beta \frac{\mu_{o}}{2 \pi}\left[\ln \left(\frac{\mathfrak{L}+\sqrt{\mathfrak{L}^{2}+P^{2}}}{P}\right) \mathfrak{L}\right. \\
& \left.+P-\sqrt{\mathfrak{L}^{2}+P^{2}}\right] \text {, for } \mathrm{DC} \\
& L_{21}=\beta \frac{\mu_{o}}{2 \pi}\left[\ln \left(\frac{\mathfrak{L}+\sqrt{\mathfrak{L}^{2}+P^{2}}}{P}\right) \mathfrak{L}\right. \\
& \left.+P-\sqrt{\mathfrak{L}^{2}+P^{2}}\right] \text {, for } f_{\text {asym }} .
\end{aligned}
$$




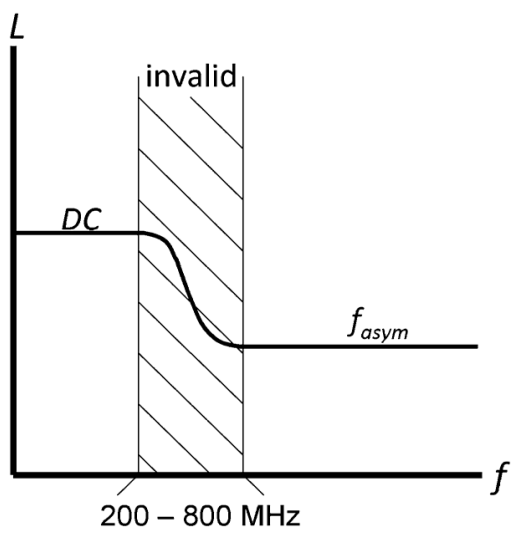

Fig. 12. Frequencies for which the closed-form inductance expressions are valid.

TABLE VII

COMPARISON OF NUMERICAL SIMULATIONS AND ANALYTIC EXPRESSIONS OF THE TSV ELECTRICAL PARAMETERS

\begin{tabular}{|c|c|c|c|}
\hline $\begin{array}{c}\text { Electrical } \\
\text { Parameters }\end{array}$ & $\begin{array}{c}\text { Numerical } \\
\text { Simulation }\end{array}$ & $\begin{array}{c}\text { Analytic } \\
\text { Expressions }\end{array}$ & \% Error \\
\hline \hline DC resistance $(\mathrm{m} \Omega)$ & 148 & 154 & 4.1 \\
\hline 1 GHz resistance $(\mathrm{m} \Omega)$ & 166 & 177 & 6.6 \\
\hline DC self inductance $(\mathrm{pH})$ & 3.9 & 3.9 & 0 \\
\hline$f_{\text {asym } \text { self inductance }(\mathrm{pH})}$ & 2.9 & 3.1 & 6.9 \\
\hline DC mutual inductance $(\mathrm{pH})$ & 1.40 & 1.32 & -5.7 \\
\hline$f_{\text {assm }}$ mutual inductance $(\mathrm{pH})$ & 1.10 & 1.08 & -1.8 \\
\hline Capacitance $(\mathrm{fF})$ & 1.43 & - & - \\
\hline
\end{tabular}

The inductance expressions are dependent on the length $\mathfrak{L}$ and radius $\mathfrak{R}$ of the TSV. The radius is replaced by the pitch $P$ for the expressions characterizing the mutual inductance $L_{21}$ between two TSVs. The $\alpha$ parameter, used to adjust the partial self-inductance, approaches unity at dc and 0.94 at high frequencies with increasing aspect ratio $\mathfrak{L} / D$, where $\mathrm{D}$ is the diameter of the TSV. The $\beta$ parameter, used to adjust the partial mutual inductance, is unity at dc and ranges between 0.49 and 0.93 at high frequencies with increasing aspect ratio [26]. Both $\alpha$ and $\beta$ are included in (12) and (13), and (14) and (15), respectively. Each parameter is determined at dc and at high frequency [26],

$$
\begin{aligned}
& \alpha= \begin{cases}1-e^{\frac{-4.3 \mathcal{E}}{D}}, & \text { if } f=\mathrm{DC} \\
0.94+0.52 e^{-10\left|\frac{\mathfrak{L}}{D-1}\right|}, & \text { if } f>f_{\text {asym }}\end{cases} \\
& \beta= \begin{cases}1, & \text { if } f=\mathrm{DC} \\
0.1535 \ln \left(\frac{\mathfrak{L}}{D}\right)+0.592, & \text { if } f>f_{\text {asym }} .\end{cases}
\end{aligned}
$$

The capacitance of a bulk 3-D via [26] is

$$
C=\alpha \beta \cdot \frac{\epsilon_{S i}}{t_{\text {diel }}+\frac{\epsilon_{S i O_{2}}}{\epsilon_{S i}} x_{d T p}} 2 \pi \Re \mathfrak{R}
$$

where $\mathfrak{R}$ is the TSV radius, $\mathfrak{L}$ is the TSV length, $t_{\text {diel }}$ is the thickness of the dielectric surrounding the $3-\mathrm{D}$ via, $x_{d T p}$ is the depletion region depth of p-type silicon, and $\epsilon_{\mathrm{SiO}_{2}}$ and $\epsilon_{\mathrm{Si}}$ are the electrical permittivity of silicon dioxide and silicon, respectively. The depletion region is dependent on the p-type silicon work function $\phi_{f_{p}}$, where $n_{i}$ is the intrinsic semiconductor concentration, $N_{A}$ is the silicon doping concentration $\left(10^{21} \mathrm{~m}^{-3}\right)$,
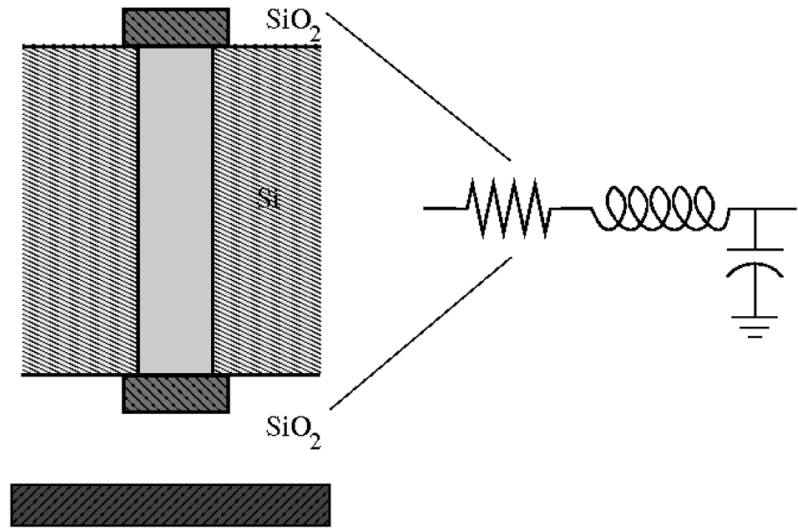

Fig. 13. Equivalent electrical model of the TSV.

and $V_{\mathrm{th}}$ is the thermal voltage. Both $x_{d T p}$ and $\phi_{f_{p}}$ are provided as (17) and (18), respectively,

$$
\begin{aligned}
x_{d T p} & =\sqrt{\frac{4 \epsilon_{S i} \phi_{f p}}{q N_{A}}} \\
\phi_{f p} & =V_{\mathrm{th}} \ln \left(\frac{N_{A}}{n_{i}}\right) .
\end{aligned}
$$

The $\alpha$ and $\beta$ fitting parameters adjust the capacitance for two physical factors: 1) the distance to the ground plane, and 2) the diminishing contribution of the upper portion of the 3-D via to the total capacitance relative to a ground plane below the via. These fitting parameters are

$$
\begin{aligned}
& \alpha=\left(-0.0351 \frac{\mathfrak{L}}{D}+1.5701\right) S_{g_{n n d_{\mu m}}^{0.0111 \frac{\mathfrak{L}}{D}}-0.1997} \\
& \beta=5.8934 D_{\mu m}^{-0.553}\left(\frac{\mathfrak{L}}{D}\right)^{-\left(0.0031 D_{\mu m}+0.43\right)} .
\end{aligned}
$$

The resistance, inductance, and capacitance expressions are compared to numerical simulations for the TSV structures used in the MITLL multiproject wafer (for 3-D via parameters, $\mathfrak{R}=$ $1 \mu \mathrm{m}, \mathfrak{L}=8.5 \mu \mathrm{m}$, and $P=5 \mu \mathrm{m}$ ) in Table VII. The equivalent electrical model of the TSV is shown in Fig. 13.

\section{APPENDIX B}

\section{Circuit Parameters to Model the Clock Skew of the 3-D CLOCK TOPOLOGIES}

The circuit parameters used to model the skew within the clock network are provided below. The dimensions of the buffer circuits at the root, leaves, and output circuitry are listed in Table VIII. Two sets of transistor widths are provided as each location is double buffered to maintain the same signal logic level. The dimensions of the ring and a single mesh are listed in Table IX. These lengths are composed of $50 \mu \mathrm{m}$ long segments, and each segment is replaced with an equivalent $\pi$ model for a line width of $4 \mu \mathrm{m}$. The source follower nMOS transistor located in the output circuitry has a length of $180 \mathrm{~nm}$, and a width of $12 \mu \mathrm{m}$. The interconnect length connecting the output pads and output circuitry to the leaves on each of the three device planes varies from 0 to $150 \mu \mathrm{m}$ depending upon the clock topology (line width of $2 \mu \mathrm{m}$ ), and is also represented by an equivalent $\pi$ model. 
TABLE VIII

Transistor Width of THE Clock Buffers at THE RoOt, LEAVES, AND OUTPUT CIRCUITRY (ALL LENGTHS ARE $180 \mathrm{~nm}$ )

\begin{tabular}{|c|c|c|c|}
\hline Buffer location & & $W_{N}(\mu \mathrm{m})$ & $W_{P}(\mu \mathrm{m})$ \\
\hline \hline \multirow{2}{*}{ Root } & Buffer 1 & 20 & 50 \\
\cline { 2 - 4 } & Buffer 2 & 54 & 136 \\
\hline \multirow{2}{*}{ Leaf } & Buffer 1 & 15 & 38 \\
\cline { 2 - 4 } & Buffer 2 & 15 & 38 \\
\hline \multirow{2}{*}{ Output circuitry } & Buffer 1 & 2.5 & 7 \\
\cline { 2 - 4 } & Buffer 2 & 2.5 & 7 \\
\hline
\end{tabular}

TABLE IX

Dimensions of LOCAL AND GLOBAL Clock RingS

\begin{tabular}{|c|c|c|}
\hline Clock topology & Length $(\mu \mathrm{m})$ & Width $(\mu \mathrm{m})$ \\
\hline \hline Global rings & 500 & 500 \\
\hline Local rings & 200 & 200 \\
\hline
\end{tabular}

\section{ACKNOWLEDGMENT}

The authors would like to thank Y. Zhu, L. Zhang, and Prof. $\mathrm{H}$. Wu of the University of Rochester for their help during testing and the MIT Lincoln Laboratories for fabricating the 3-D test circuit.

\section{REFERENCES}

[1] Clock Distribution Networks in VLSI Circuits and Systems, E. G. Friedman, Ed.. Piscataway, NJ: IEEE Press, 1995.

[2] E. G. Friedman, "Clock distribution networks in synchronous digital integrated circuits," Proc. IEEE, vol. 89, no. 5, pp. 665-692, May 2001.

[3] W. Bailey and B. J. Benschneider, "Clocking design and analysis for a 600-MHz alpha microprocessor," IEEE J. Solid-State Circuits, vol. 22, no. 11, pp. 1627-1633, Nov. 1998.

[4] T. Xanthopoulos, D. W. Bailey, A. K. Gangwar, M. K. Gowan, A. K. Jain, and B. K. Prewitt, "The design and analysis of the clock distribution network for a $1.2 \mathrm{GHz}$ alpha microprocessor," in Proc. IEEE Int. Solid-State Circuits Conf., 2001, pp. 402-403.

[5] ScienceDaily, "3-D computer processor: "Rochester Cube" points way to more powerful chip designs," Sep. 17, 2008. [Online]. Available: www.sciencedaily.com/releases/2008/09/080915105733.htm

[6] V. F. Pavlidis, I. Savidis, and E. G. Friedman, "Clock distribution networks for 3-D integrated circuits," in Proc. IEEE Int. Conf. Custom Integr. Circuits, 2008, pp. 651-654.

[7] V. F. Pavlidis, I. Savidis, and E. G. Friedman, "Clock distribution architectures for 3-D SOI integrated circuits," in Proc. IEEE Int. Silicon-on-Insulator Conf., 2008, pp. 111-112.

[8] D. L. Lewis and H.-H. S. Lee, "A scan-island based design enabling pre-bond testability in die-stacked microprocessor," in Proc. IEEE Int. Test Conf., 2007, pp. 21.2.1-21.2.8.

[9] W. R. Davis, J. Wilson, S. Mick, J. Xu, H. Hua, C. Mineo, A. M. Sule, M. Steer, and P. D. Franzon, "Demystifying 3D ICs: The pros and cons of going vertical," IEEE Des. Test Comput., vol. 22, no. 6, pp. 498-510, Nov./Dec. 2005

[10] J. Minz, X. Zhao, and S. K. Lim, "Buffered clock tree synthesis for 3D ICs under thermal variations," in Proc. IEEE Int. Asia South Pacific Des. Autom. Conf., 2008, pp. 504-509.

[11] M. Mondal, A. J. Ricketts, S. Kirolos, T. Ragheb, G. Link, N. Viyaykrishnan, and Y. Massoud, "Thermally robust clocking schemes for 3D integrated circuits," in Proc. IEEE Int. Conf. Des., Autom. Test Euro. (DATE), 2007, pp. 1206-1211.

[12] V. Arunachalam and W. Burleson, "Low-power clock distribution in a multilayer core 3D microprocessor," in Proc. ACM/IEEE Int. Great Lakes Symp. VLSI, 2008, pp. 429-434.

[13] “MITLL Low-Power FDSOI CMOS Process Design Guide, ” MIT Lincoln Laboratories, Cambridge, MA, 2006.

[14] J. A. Burns, B. F. Aull, C. K. Chen, C.-L. Chen, C. L. Keast, J. M. Knecht, V. Suntharalingam, K. Warner, P. W. Wyatt, and D.-R. W. Yost, "A wafer-scale 3-D circuit integration technology," IEEE Trans. Electron Devices, vol. 53, no. 10, pp. 2507-2515, Oct. 2006.
[15] W. Cui, H. Chen, and Y. Han, "VLSI implemenation of universal random number generator," in Proc. IEEE Asia-Pacific Conf. Circuits Syst., 2002, pp. 465-470.

[16] M. Popovich, A. V. Mezhiba, and E. G. Friedman, Power Distribution Networks With On-Chip Decoupling Capacitors. New York: Springer Verlag, 2008

[17] N. Hedenstiema and K. O. Jeppson, "CMOS circuit speed and buffer optimization," IEEE Trans. Comput.-Aided Des. Integr. Circuits Syst., vol. 6, no. 2, pp. 270-281, Mar. 1987.

[18] B. S. Cherkauer and E. G. Friedman, "A unified design methodology for CMOS tapered buffers," IEEE Trans. Very Large Scale Integr. (VLSI) Syst., vol. 3, no. 1, pp. 99-111, Mar. 1995.

[19] H. B. Bakoglu, Circuits, Interconnections, and Packaging for VLSI. Boston, MA: Addison-Wesley, 1990.

[20] M. W. Newman, S. Muthukumar, M. Schuelein, T. Dambrauskas, P. A. Dunaway, J. M. Jordan, S. Kulkarni, C. D. Linde, T. A. Opheim, R. A. Stingel, W. Wormag, L. A. Topic, and J. M. Swan, "Fabrication and electrical characterization of 3D vertical interconnects," in Proc. IEEE Int. Electron. Components Technol. Conf., 2006, pp. 394-398.

[21] P. Dixit and J. Miao, "Fabrication of high aspect ratio $35 \mu \mathrm{m}$ pitch interconnects for next generation 3-D wafer level packaging by throughwafer copper electroplating," in Proc. IEEE Int. Electron. Components Technol. Conf., 2006, pp. 388-393.

[22] K. Sugahara, T. Nishimura, S. Kusunoki, Y. Akasaka, and H. Nakata, "SOI/SOI/bulk-Si triple-level structure for three-dimensional devices," IEEE Electron Device Lett., vol. EDL-7, no. 3, pp. 193-194, Mar. 1986.

[23] A. Fan, A. Rahman, and R. Reif, "Copper wafer bonding," Electrochem. Solid-State Lett., vol. 2, no. 10, pp. 534-536, Oct. 1999.

[24] A. W. Topol, D. C. Tulipe, L. L. Shi, D. J. Frank, K. Bernstein, S. E. Stein, A. Kumar, G. U. Singco, A. M. Young, K. W. Guarini, and M. Ieong, "Three-dimensional integrated circuits," IBM J. Res. Development, vol. 50, no. 4/5, pp. 491-506, 2006.

[25] I. Savidis and E. G. Friedman, "Electrical modeling and characterization of 3-D vias," in Proc. IEEE Int. Symp. Circuits Syst., 2008, pp. 784-787.

[26] I. Savidis and E. G. Friedman, "Closed-form expressions of 3-D via resistance, inductance, and capacitance," IEEE Trans. Electron Devices, vol. 56, no. 9, pp. 1873-1881, Sep. 2009.

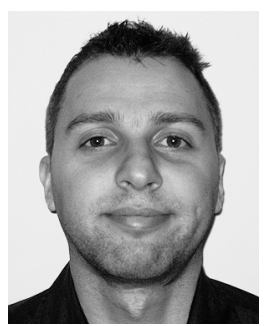

Vasilis F. Pavlidis (M'09) received the B.S. and M.Eng. degrees in electrical and computer engineering from the Democritus University of Thrace, Xanthi, Greece, in 2000 and 2002, respectively, and the M.Sc. and Ph.D. degrees from the Department of Electrical and Computer Engineering, University of Rochester, Rochester, NY, in 2003 and 2008, respectively.

He is currently a post-doctoral researcher with the Integrated Systems Laboratory, EPFL, Lausanne, Switzerland. From 2000 to 2002, he was with INTRACOM S.A., Athens, Greece. In summer 2007, he was with Synopsys Inc., Mountain View, CA. His current research interests include the area of interconnect modeling, 3-D integration, networks-on-chip, and related design issues in VLSI.

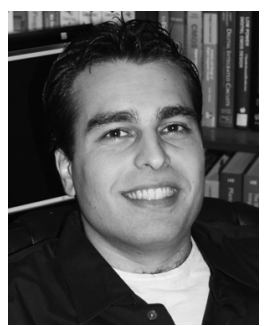

Ioannis Savidis received the B.S.E. degree in electrical and computer engineering and biomedical engineering from Duke University, Durham, NC, in 2005, and the M.Sc. degree in electrical and computer engineering from the University of Rochester, Rochester, $\mathrm{NY}$, in 2007, where he is currently pursuing the Ph.D. degree in electrical and computer engineering under the guidance of Prof. E. G. Friedman.

He interned at Freescale Semiconductor Corporation, Austin, TX, during the summers of 2006 and 2007, where he worked on the electrical characterization and modeling of interplane 3-D vias. During the summers of 2008-2010, he interned at T. J. Watson Research Center, IBM, Yorktown Heights, NY, where he worked on electrical characterization and modeling, reticle design, and DC and high frequency electrical measurements of test vehicles implementing various interplane 3-D via topologies. His research interests include analysis, modeling, and design methodologies for high performance digital and mixed signal integrated circuits, with an emphasis on electrical and thermal modeling and characterization, signal and power integrity, and power and clock delivery for 3-D stacking technologies. 


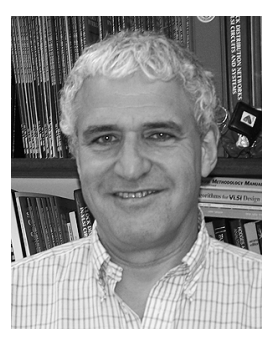

Eby G. Friedman (F'00) received the B.S. degree from Lafayette College, Easton, PA, in 1979, and the M.S. and Ph.D. degrees from the University of California, Irvine, in 1981 and 1989, respectively, all in electrical engineering.

From 1979 to 1991, he was with Hughes Aircraft Company, rising to the position of Manager of the Signal Processing Design and Test Department, responsible for the design and test of high performance digital and analog ICs. He has been with the Department of Electrical and Computer Engineering, University of Rochester, Rochester, NY, since 1991, where he is a Distinguishe Professor and the Director of the High Performance VLSI/IC Design and Analysis Laboratory. He is also a Visiting Professor at the Technion-Israel Institute of Technology, Haifa. His current research and teaching interests include high performance synchronous digital and mixed-signal microelectronic design and analysis with application to high speed portable processors and low power wireless communications. He is the author of about 400 papers and book chapters, numerous patents, and the author or editor of 14 books in the fields of high speed and low power CMOS design techniques, high speed interconnect, and the theory and application of synchronous clock and power distribution networks.

Dr. Friedman is the Regional Editor of the Journal of Circuits, Systems and Computers, a Member of the editorial boards of the Analog Integrated Circuits and Signal Processing, Microelectronics Journal, Journal of Low Power Electronics, Journal of Low Power Electronics and Applications, and Journal of VLSI Signal Processing. He is the Chair of the IEEE TRANSACTIONS ON Very LARGe SCALE INTEGRATION (VLSI) SYSTEMS steering committee, and a Member of the technical program committee of a number of conferences. He previously was the Editor-in-Chief of the IEEE TRANSACTIONS ON VERY LARGE SCALE INTEGRATION (VLSI) SYSTEMS, a Member of the editorial board of the ProceEdings of THE IEEE and IEEE TRANSACTIONS ON CirCUITS AND Systems II: ANAlog AND Digital Signal Processing, a Member of the Circuits and Systems (CAS) Society Board of Governors, Program and Technical Chair of several IEEE conferences. He was a recipient of the University of Rochester Graduate Teaching Award and College of Engineering Teaching Excellence Award. Dr. Friedman is a Senior Fulbright Fellow. 